\title{
Successful Surgical and Medical Management of Cesarean Scar Pregnancy in 2 Patients
}

\author{
Hina Javaid, MD, ${ }^{1,2}$ and Mostafa I. Abuzeid, MD, FACOG, FRCOG ${ }^{2-4}$
}

\begin{abstract}
Background: Cesarean scar pregnancy (CSP), once a rare entity, is on the rise due because of an increase in the cesarean section rate worldwide. Currently, there is no standard protocol available for managing CSP. To contribute to the existing literature, this article presents the current authors' experience with 2 cases of CSP that were treated successfully with two different modalities. Cases: Case 1: A 34-year-old, gravida 2, para 1, was diagnosed with a CSP on initial transvaginal ultrasound (TVUS) scan at 6 weeks of gestation. Aspiration of the gestational sac and a local injection of methotrexate was performed. After 2 weeks, the gestational sac increased in size with thinning of the CS scar $(1 \mathrm{~mm})$, and plateauing of the $\beta$-human chorionic gonadotropin ( $\beta$-hCG) occurred. Laparoscopic excision of the CSP and myometrial repair resulted in resolution. Case 2: A 31-year-old, gravida 3, para 1 , achieved pregnancy after a frozen-thawed embryo transfer cycle. A TVUS scan, performed at 6 weeks of gestation showed a CSP. The patient's B-hCG level was $310 \mathrm{mIU} / \mathrm{mL}$. Systemic methotrexate was administered intramuscularly. The patient's $B$-hCG on days 4 and 7 was 260 and 252, respectively. A repeat TVUS on day 7 showed a resolving gestational sac. A second dose of methotrexate resulted in complete resolution of the CSP. Results: The treatments (aspiration, methotrexate, and laparoscopic excision for Case 1, and methotrexate for Case 2) enabled resolution of the CSPs of these 2 patients. Conclusions: Various treatment modalities have been described for managing CSP with varied levels of success. When local injection of methotrexate into the gestational sac of CSP is unsuccessful, laparoscopic removal is safe and effective. Moreover, in the presence of low levels of $\beta$-hCG, treatment with systemic methotrexate is usually successful. ( $\mathrm{J}$ GYNECOL SURG 30:168)
\end{abstract}

\section{Introduction}

$\mathbf{C}$ ESAREAN SCAR PREGNANCY (CSP) is a pregnancy occurring in the uterine scar of a prior cesarean section. CSP is a long-term complication of a prior cesarean delivery, and the numbers of cases reported are increasing every year. This rise appears to be secondary to increasing awareness and an increase in the cesarean-section rate across the world. ${ }^{1,2}$ Given this increase, there seems to be a significant underreporting of CSP as evidenced by the small number of cases reported in the United States. The incidence of CSP has been estimated to range between $1 / 1800$ and $1 / 2500$ of all cesarean deliveries performed. ${ }^{3,4} \mathrm{CSP}$ constitutes $6.1 \%$ of all ectopic pregnancies, in patients with histories of at least one cesarean delivery. ${ }^{3,5,6}$ The etiology and pathophysiology of CSP is still under investigation, and a satisfactory explanation cannot be provided at this time. However, one of the most-considered pathophysiol- ogies is that implantation of the embryo into the uterine wall occurs through an internal dehiscence of the scar or through a small pathway from the endometrial canal to the scar tissue..$^{7-10}$

CSP is often misdiagnosed as a cervical ectopic pregnancy, an incomplete abortion, or a lower intrauterine pregnancy. For instance, the diagnosis of CSP was missed in $13.6 \%$ of the reported cases. ${ }^{11}$ This delay in the diagnosis and subsequent management can lead to potentially life-threatening complications. ${ }^{3,8,12,13}$ Moreover, the overall complications of different treatment regimens are reported to be as high as $44.1 \% .^{11}$ This indicates the need for a management protocol with the lowest complication rate and the highest success rate. A review of the literature revealed that there is no standard protocol available for managing CSP. To contribute to the existing literature, the current authors present their experience with 2 cases of CSP who were treated successfully with two different management modalities.

\footnotetext{
${ }^{1}$ Department of Obstetrics and Gynecology, Hurley Medical Center, Flint MI.

${ }^{2}$ Department of Obstetrics and Gynecology and Reproductive Biology, Michigan State University, College of Human Medicine, Flint Campus, Flint MI.

${ }^{3}$ Division of Reproductive Endocrinology and Infertility, Department of Obstetrics and Gynecology, Hurley Medical Center, Flint MI.

${ }^{4}$ IVF Michigan PC, Flint and Rochester Hills, MI.
} 


\section{Cases}

Case 1

A 34-year-old Caucasian woman, gravida 2, para 1, presented for a routine obstetrical transvaginal ultrasound (TVUS) at 9-weeks of gestation. She had a positive home pregnancy test after missing a period a few weeks prior. Her pregnancy had been uneventful other than some mild spotting at the gestational age of 7 weeks for which the patient did not seek medical attention. She had a prior history of a low transverse cesarean section 1 year prior, which was performed after a failed induction of labor. The dating ultrasound showed a gestational sac in the cervical canal. A possibility of cervical ectopic pregnancy versus an incomplete abortion was considered. A quantitative assay of her level of B-subunit of human chorionic gonadotrophin (BhCG) was $9084 \mathrm{mIU} / \mathrm{mL}$. This $\mathrm{B}$-hCG 2 days later was $10328 \mathrm{mIU} / \mathrm{mL}$. A second ultrasound (Fig. 1 A and B) showed a gestational sac with a yolk sac in the cervical canal, confirming the diagnosis of either a cervical ectopic pregnancy or a CSP. The patient was offered options of either undergoing a systemic injection of methotrexate or an aspiration of the pregnancy, followed by local injection of methotrexate. She chose the latter option. The next day, the patient underwent TVUS-guided aspiration of the gestational sac followed by a local injection of $77.5 \mathrm{mg}\left(50 \mathrm{mg} / \mathrm{m}^{2}\right)$ of methotrexate. She tolerated the procedure well and went home on the same day. TVUS scanning performed 1 week after treatment showed a collapse of the gestational sac (Fig. 2 ). Repeat testing of her levels of $\beta$-hCG at days $4,6,7,10$, 13, and 17 after the procedure were 16911, 14420, 11502, $8653,8196,7426 \mathrm{mIU} / \mathrm{mL}$, respectively. Plateauing of $\beta-\mathrm{hCG}$ levels was observed. A repeat TVUS at that time showed reaccumulated fluid in the gestational sac with a yolk sac located in the CS scar (Fig. 3). The myometrium of the CS scar was very thin over the gestational sac $(1 \mathrm{~mm})$. The patient was advised that the best treatment option was to undergo surgery to prevent a possible rupture of the CSP. Diagnostic and operative laparoscopy with a possible laparotomy was arranged. During laparoscopy, it was observed that the bladder
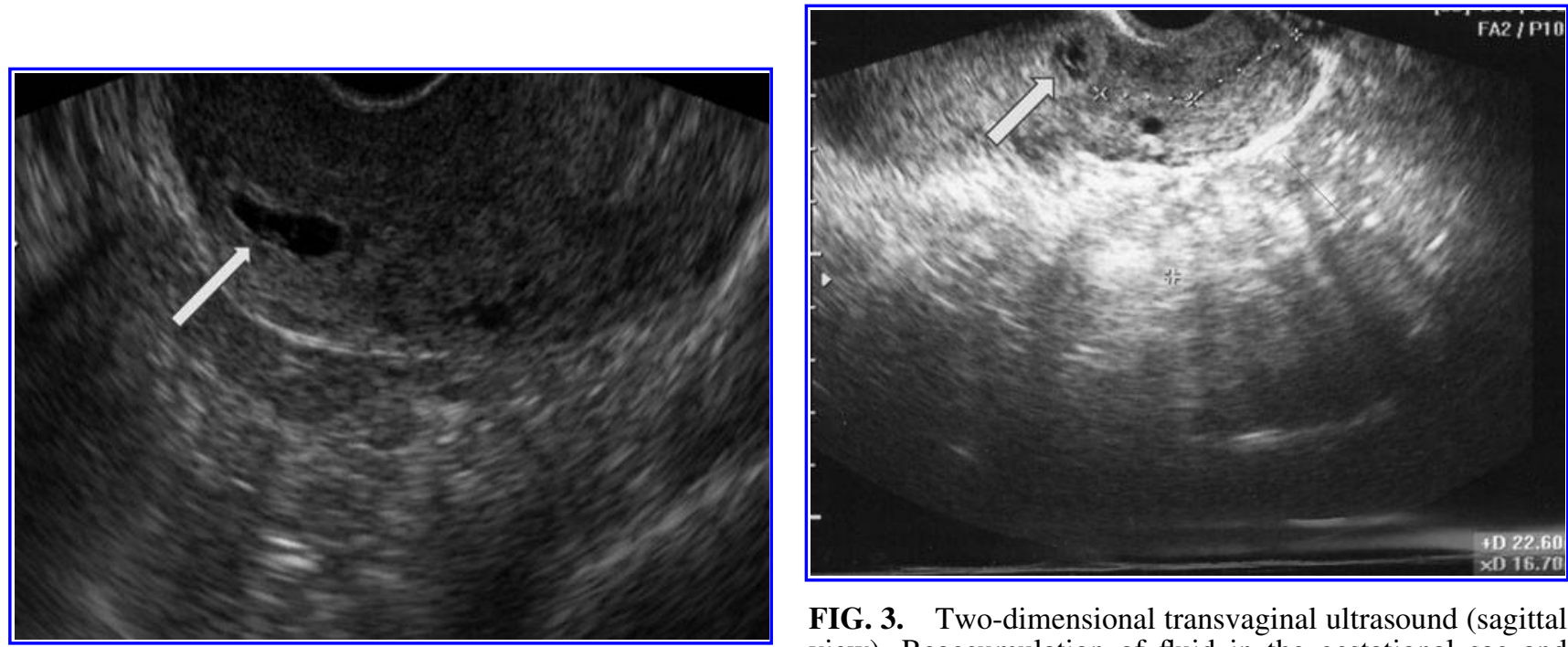

FIG. 3. Two-dimensional transvaginal ultrasound (sagittal view). Reaccumulation of fluid in the gestational sac and

FIG. 1. Two-dimensional transvaginal ultrasound (sagittal view). Gestational sac and yolk sac seen at the region of internal os (arrow).

sarean scar noted 2 weeks post aspiration and intragestational methotrexate. 


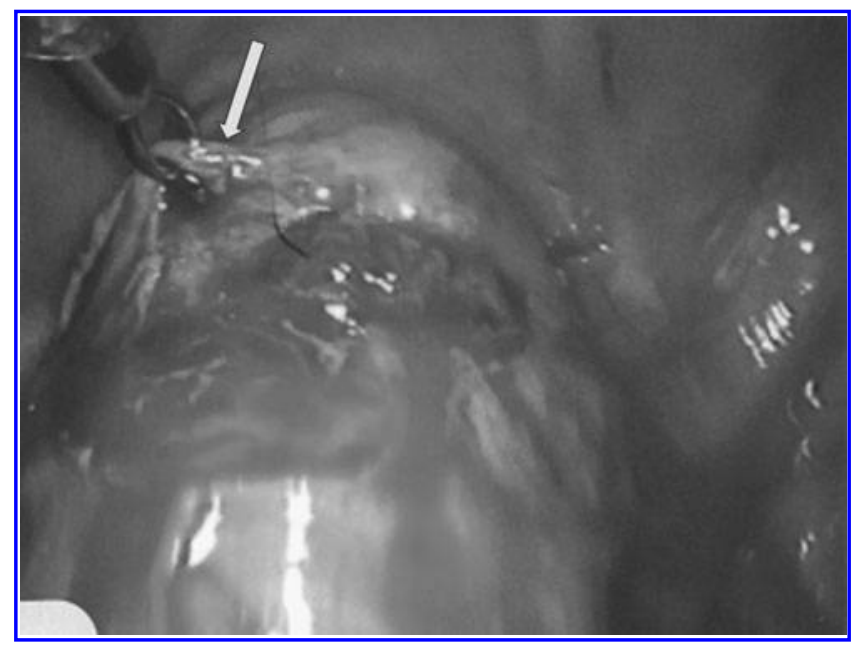

FIG. 4. Bladder adhered to the lower uterine segment over the prior cesarean section scar (arrow).

(IVF-FET) with an estrogen- and progesterone-stimulated cycle. Two blastocysts were transferred without any complications. This patient had a prior history of a successful IVF pregnancy (for male-factor infertility) that was delivered at term via a low transverse cesarean section 2.5 years ago. Her first quantitative B-hCG level 2 weeks post IVFFET was $246 \mathrm{mIU} / \mathrm{mL}$. She had brownish spotting and mild cramping 15 days after the IVF-FET; this symptom subsided in 2 days. A repeat B-HCG test in another 2 weeks showed a level of $310 \mathrm{mIU} / \mathrm{mL}$. TVUS was performed, which showed a gestational sac in the cesarean scar with an otherwise normal uterine cavity. (Fig. $7 \mathrm{~A}$ and B). Only a small gestational sac and yolk sac were noted, but no fetal pole was seen. Various treatment modalities were discussed with the patient, and she chose systemic methotrexate therapy. She was given $113.5 \mathrm{mg}$ $\left(50 \mathrm{mg} / \mathrm{m}^{2}\right)$ of methotrexate intramuscularly (IM) the next day. Post methotrexate, this patient started having vaginal bleeding for 6 days. Her B-HCG levels on days 4 and 7 were 260 and $252 \mathrm{mIU} / \mathrm{mL}$, respectively. A repeat TVUS on day 7 showed an empty uterus (Fig. 8A and B). However, a decision was made to administer a second dose of methotrexate secondary to her plateauing $\beta$-HCG levels. She was given $113.5 \mathrm{mg}\left(50 \mathrm{mg} / \mathrm{m}^{2}\right)$ of methotrexate IM again. Her $\beta-\mathrm{HCG}$ levels on days 4 and 7 were 83 and $51 \mathrm{mIU} / \mathrm{mL}$, respectively. Her B-HCG levels normalized within 1 month of administration of the first dose of methotrexate.

\section{Results}

The treatments (aspiration, methotrexate, and laparoscopic excision for Case 1, and methotrexate for Case 2) enabled resolution of the CSPs of these 2 patients.

\section{Discussion}

CSP represents a diagnostic and management dilemma. Delayed diagnosis and management can lead to life-threatening complications. . $^{3,8,11,13}$ The increasing rate of cesarean deliveries, a higher index of suspicion secondary to mounting awareness, and better diagnostic studies such as high-frequency TVUS can possibly explain the recent increase of cases described in the literature. $3,5,14$

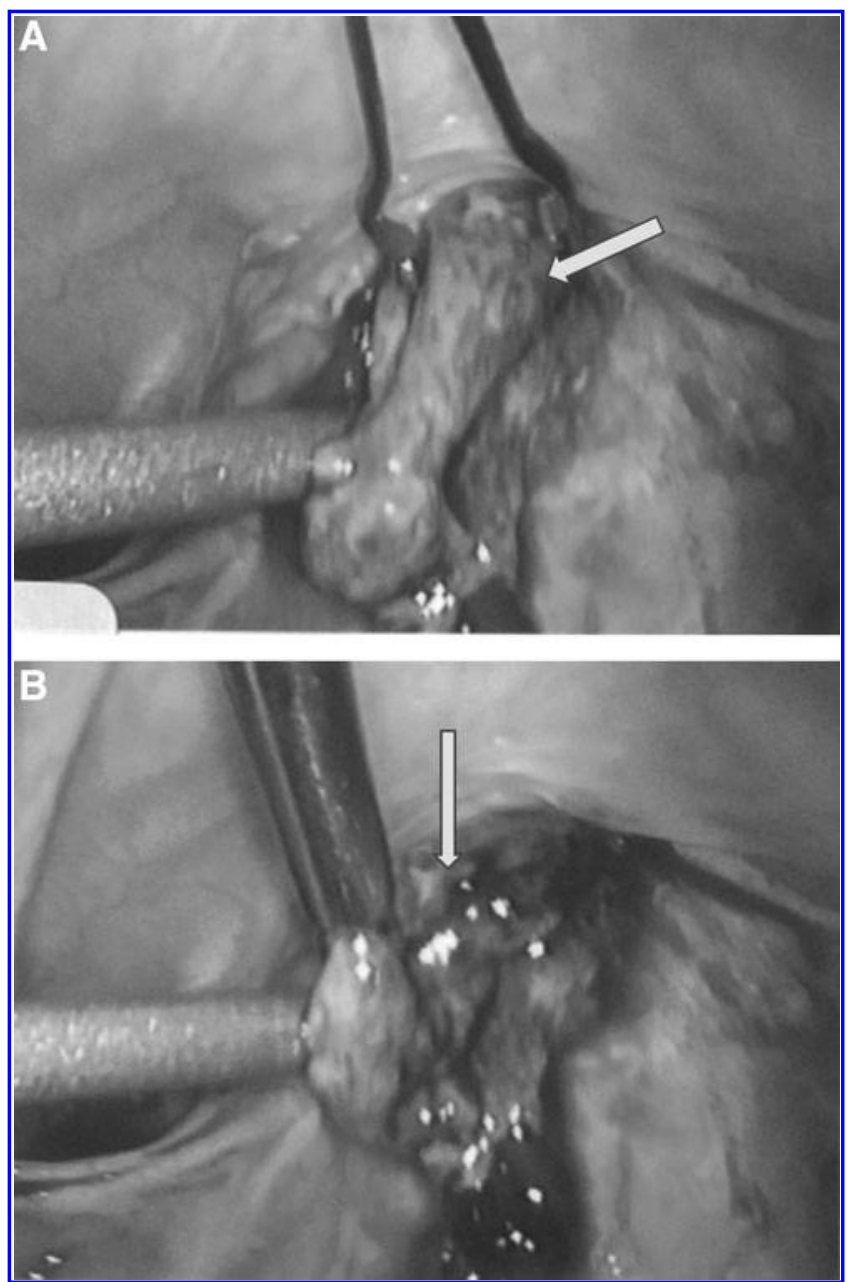

FIG. 5. (A) After dissecting bladder flap, the ectopic gestation (arrow) is being manipulated by grasping forceps. (B) Removal of ectopic gestation in progress (arrow).

Many management modalities have been described in the literature. In a review article, Timor-Tritsch et al. describe a combined modality with the lowest complication rate that was based on a review of literature and their own experience. ${ }^{11}$ These researchers recommended local intragestational and

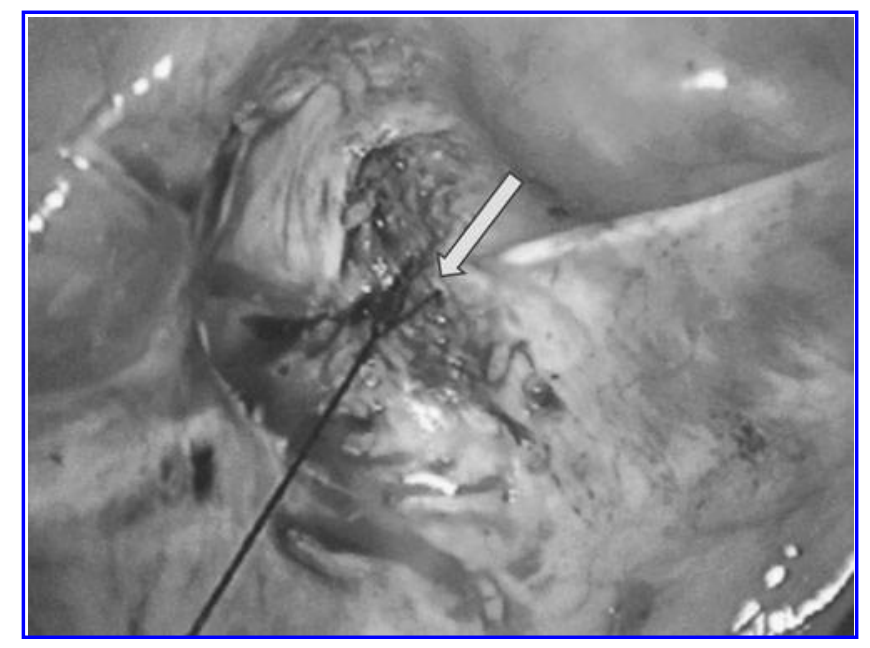

FIG. 6. Closure of uterine defect (arrow). 

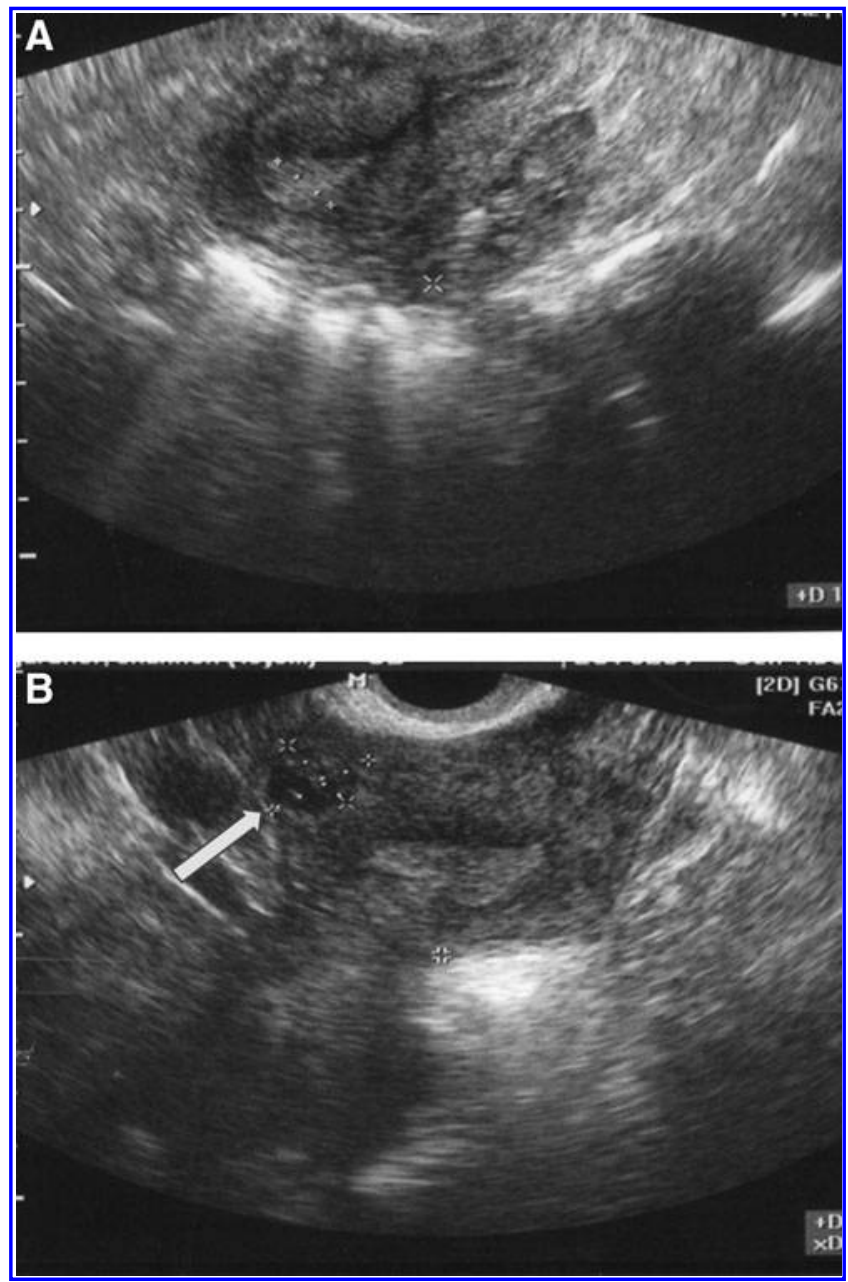

FIG. 7. (A) Two-dimensional (2D) transvaginal ultrasound (TVUS; sagittal view) thickened endometrial stripe. No pregnancy sac was identified. (B) 2D TVUS (transverse view). Small gestational sac and yolk sac at the region of the cesarean scar (arrow).

systemic methotrexate therapy; this was reported to produce a complication rate as low as $5.3 \%$ in the cases that were treated. The downside of this treatment is a longer return to baseline and a lengthy follow-up, which is up to a minimum of 3 months. This might not be suitable for a noncompliant patient or one who will not be able to come back for frequent follow-up visits. In Case 1, a local injection of methotrexate and gestational sac aspiration resulted in plateauing of the patient's B-hCG levels. Reaccumulation of fluid in the gestational sac and marked thinning of the ceserean scar predisposed the CSP to a high risk of impending rupture; hence, laparoscopic surgery was performed without any complications and a quick return of $\mathrm{B}-\mathrm{hCG}$ to baseline was observed.

Vial et al. ${ }^{15}$ has described two distinct types of CSP: (1) Superficial implantation in the scar with progression of the pregnancy toward the uterine cavity; and (2) deeper implantation in a cesarean-scar defect with pregnancy progression outside the uterine cavity. One of the surgical treatments with the lowest complication rate described was hysteroscopic resection (14.7\%). ${ }^{11}$ A literature review showed that this modality might be one of the best available surgical man-
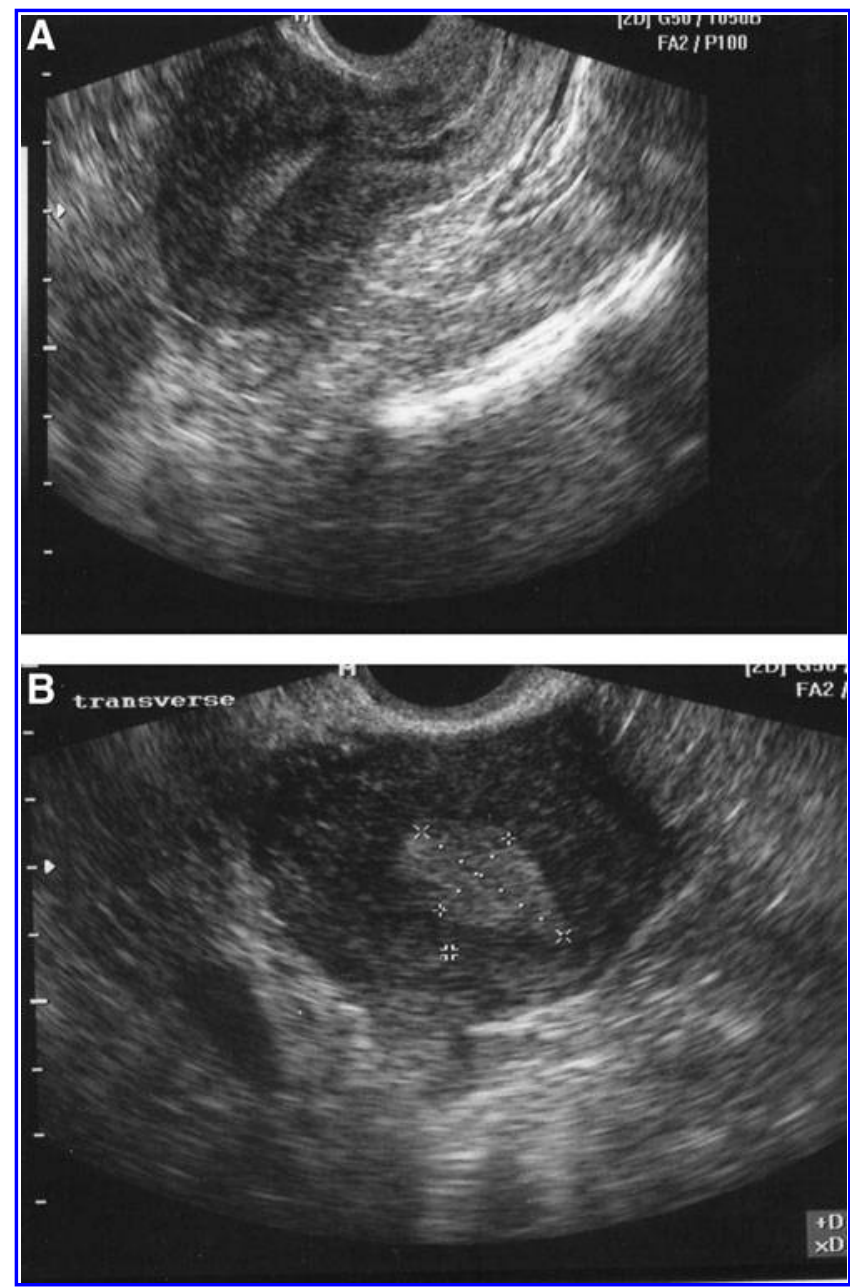

FIG. 8. (A) Two-dimensional (2D) transvaginal ultrasound (TVUS; sagittal view) showing resolution of cesarean scar ectopic pregnancy 1 week post systemic methotrexate. (B) 2D TVUS (transverse view). Resolution of cesarean scar ectopic pregnancy 1 week post systemic methotrexate.

agements for a CSP that projects inside the uterine cavity, because this approach can enable direct visualization of the vessels and subsequent coagulation, as the CSP is resected with a resectoscope. ${ }^{16-24}$ However, hysteroscopic resection might not be the best choice when the pregnancy is implanted deeper in the cesarean scar and tends to progress toward the bladder. In case 1 , the TVUS revealed a very thin myometrium of $1-2 \mathrm{~mm}$ overlying the scar and a CSP in close proximity to the bladder. The decision was made to perform laparoscopy in order to dissect the bladder away from the CSP under direct visualization and hence reduce the risk of injury. This would have been very challenging if a hysteroscopic resection had been performed. This concept has also been supported by other researchers. ${ }^{10,25}$ However, it remains undisputed that both operative laparoscopy and hysteroscopy are heavily dependent on a surgeon's expertise. Systemic methotrexate is a modality with one of the highest complication rates (i.e., $62.1 \%$ ). ${ }^{11}$ The literature review also showed that, if $\beta$-hCG was $<5000$ and the gestational age was $<8$ weeks, the chance of success was higher..$^{3,5-7,26}$ In the experience of the current authors, Case 2 was diagnosed very 
early in her pregnancy secondary to close follow-up after her IVF-FET. She was treated with 2 doses of systemic methotrexate 1 week apart, which resulted in a prompt resolution without any complications (1 month).

\section{Conclusions}

An earlier diagnosis leads to a better outcome. Various treatment modalities have been described for managing CSP, with varied levels of success, but the optimal management and a standardized protocol remains to be determined. The goal of therapy should be preservation of fertility, reduction of life-threatening complications, and maintaining the patient's quality of life. In the current author's experience, when local injection of methotrexate in the gestational sac is not successful, laparoscopic removal of CSP is safe and effective. In the presence of low levels of $\beta$-hCG, treatment with systemic methotrexate is usually successful.

\section{Acknowledgments}

The authors would like to thank Cheryl Anderson, AAS, IVF, Michigan, P.C., for her assistance in the preparation of this article.

\section{Disclosure Statement}

Hina Javaid, MD, has no competing financial interests to disclose, nor does she have any commercial associations that might creat a conflict of interest to disclose.

Mostafa Abuzeid, MD, has no competing financial interests to disclose, nor does he have any commercial associations that might creat a conflict of interest to disclose.

\section{References}

1. Brennan DJ, Robson MS, Murphy M, O'Herlihy C. Comparative analysis of international cesarean delivery rates using 10-group classification identifies significant variation in spontaneous labor. Am J Obstet Gynecol 2009;201: 308.e1-8.

2. Menacker F, Hamilton BE. Recent trends in cesarean delivery in the United States. NCHS Data Brief 2010;35:1.

3. Rotas MA, Haberman S, Levgur M. Cesarean scar ectopic pregnancies: Etiology, diagnosis and management. Obstet Gynecol 2006;107:1373.

4. Timor-Tritsch IE, Monteagudo A, Arslan AA, Santos R, Tsymbal T. The diagnosis, treatment and follow-up of cesarean scar pregnancy. Am J Obstet Gynecol 2012;207: 44.e1-13.

5. Jurkovic D, Hillaby K, Woelfer B, Lawrence S, Salim R, Elson CJ. First trimester diagnosis and management of pregnancies implanted into the lower uterine segment cesarean section scar. Ultrasound Obstet Gynecol 2003;21:220.

6. Seow KM, Huang LW, Lin YH, Lin MY, Tsai YL, Hwang JL. Cesarean scar pregnancy: Issues in management. Ultrasound Obstet Gynecol 2004;23:247.

7. Maymon R, Halperin R, Mendlovic S, Schneider D, Herman A. Ectopic pregnancies in a cesarean scar: Review of the medical approach to an iatrogenic complication. $\underline{\text { Hum }}$ Reprod Update 2004; 10:515.

8. Ash A, Smith A, Maxwell D. Cesarean scar pregnancy. BJOG 2007;114:253.

9. Tan G, Chong Y, Biswas A. Cesarean scar pregnancy: A diagnosis to consider carefully in patients with risk factors. Ann Acad Singapore 2005;34:216.
10. Litwicka K, Greco E. Cesarean scar pregnancy: A review of management options. Curr Opin Obstet Gynecol 2011; 23:415.

11. Timor-Tritsch IE, Monteagudo A. Unforeseen consequences of the increasing rate of cesarean deliveries: Early placenta accreta and cesarean scar pregnancy. A review. Am J Obstet Gynecol 2012;207:14.

12. Sadeghi H, Rutherford T, Rackow BW, Campbell KH, et al. Cesarean scar ectopic pregnancy: Case series and review of the literature. Am J Perinatol 2010;27:111.

13. Maymon R, Halperin R, Mendlovic S, Schneider D, Herman A. Ectopic pregnancies in a caesarean scar: Review of the medical approach to an iatrogenic complication. $\underline{\text { Hum }}$ Reprod Update 2004;10:515.

14. Leitch CR, Walker JJ. The rise in cesarean section rate: The same indications but a lower threshold. Obstet Gynecol Surv 1999;54:19.

15. Vial Y, Petignat P, Hohlfeld P. Pregnancy in a cesarean scar. Ultrasound Obstet Gynecol 2000;16:592.

16. Wang CJ, Tsai F, Chen C, Chao A. Hysteroscopic management of heterotopic cesarean scar pregnancy. Fertil Steril 2010;94:1529.e15-8.

17. Chueh HY, Cheng PJ, Wang CW, Shaw SW, Lee CL, Soong YK. Ectopic twin pregnancy in cesarean scar after in vitro fertilization/embryo transfer: case report. Fertil Steril 2008;90:2009.e19-21.

18. $\overline{\mathrm{He}} \mathrm{M}$, Chen MH, Xie HZ, et al. Transvaginal removal of ectopic pregnancy tissue and repair of uterine defect for caesarean scar pregnancy. BJOG 2011;118:1136.

19. Fernandez $H$. Isthmic pregnancy located in a previous caesarean section scar treated with methotrexate: A case report. Gynecol Obstet Fertil 2005;33:772.

20. Kucera E, Krepelka P, Krofta L, Feyereisl J. Cesarean scar ectopic pregnancy. Ceska Gynekol 2007;72:207.

21. Yang Q, Piao S, Wang G, Wang Y, Liu C. Hysteroscopic surgery of ectopic pregnancy in the cesarean section scar. J Minim Invasive Gynecol 2009;16:432.

22. Deans R, Abbott J. Hysteroscopic management of cesarean scar ectopic pregnancy. Fertil Steril 2010;93:1735.

23. Wang CJ, Chao AS, Yuen LT, Wang CW, Soong YK, Lee CL. Endoscopic management of cesarean scar pregnancy. Fertil Steril 2006;85:494.e1-4.

24. Annappa M, Tripathi L, Mahendran M. Caesarean section scar ectopic pregnancy presenting as a fibroid. $\mathrm{J}$ Obstet Gynaecol 2009;29:774.

25. Chao A, Wang TH, Wang CJ, Lee CL, Chao AS. Hysteroscopic management of cesarean scar pregnancy after unsuccessful methotrexate treatment. J Minim Invasive Gynecol 2005;12:374.

26. Ravhon A, Ben-Chetrit A, Rabinowitz R, Neuman M, Beller U. Successful methotrexate treatment of a viable pregnancy within a thin uterine scar. Br J Obstet Gynaecol 1997; 104:628.

Address correspondence to: Mostafa Abuzeid, MD, FACOG, FRCOG Division of Reproductive Endocrinology and Infertility Department of Obstetrics and Gynecology Hurley Medical Center Two Hurley Plaza Suite 209

Flint, MI 48503

E-mail: reprod1@hurleymc.com 